\title{
Some Identities on the High-Order $q$-Euler Numbers and Polynomials with Weight 0
}

\author{
Jongsung Choi, ${ }^{1}$ Hyun-Mee Kim, ${ }^{2}$ and Young-Hee Kim ${ }^{1}$ \\ ${ }^{1}$ Division of General Education-Mathematics, Kwangwoon University, Seoul 139-701, Republic of Korea \\ ${ }^{2}$ Department of General Education-Mathematics, Kookmin University, Seoul 136-702, Republic of Korea
}

Correspondence should be addressed to Young-Hee Kim; yhkim@kw.ac.kr

Received 7 February 2013; Accepted 2 April 2013

Academic Editor: Chun-Gang Zhu

Copyright (c) 2013 Jongsung Choi et al. This is an open access article distributed under the Creative Commons Attribution License, which permits unrestricted use, distribution, and reproduction in any medium, provided the original work is properly cited.

We construct the $N$ th order nonlinear ordinary differential equation related to the generating function of $q$-Euler numbers with weight 0 . From this, we derive some identities on $q$-Euler numbers and polynomials of higher order with weight 0 .

\section{Introduction}

As a well-known definition, the Euler polynomial $E_{n}(x)$ is given by

$$
\frac{2}{e^{t}+1} e^{x t}=\sum_{n=0}^{\infty} E_{n}(x) \frac{t^{n}}{n !}
$$

In the special case, $x=0, E_{n}(0)=E_{n}$ is the $n$th Euler number.

From (1), we note that

$$
E_{0}=1, \quad(E+1)^{n}+E_{n}=0, \quad \text { if } n>0,
$$

with the usual convention of replacing $E^{n}$ by $E_{n}$ (see [1-16]).

In the viewpoint of the $q$-extension of (1) and (2), let us consider the following $q$-Euler number and polynomial:

$$
\begin{gathered}
\frac{2}{q e^{t}+1} e^{x t}=\sum_{n=0}^{\infty} \widetilde{E}_{n, q}(x) \frac{t^{n}}{n !}, \\
\widetilde{E}_{0, q}=\frac{2}{1+q}, \quad q\left(\widetilde{E}_{q}+1\right)^{n}+\widetilde{E}_{n, q}=0, \quad \text { if } n>0,
\end{gathered}
$$

with the usual convention of replacing $\widetilde{E}_{q}^{n}$ by $\widetilde{E}_{n, q}$.

Equation (3) is called the generating function of $q$-Euler polynomial with weight 0 . In the case $x=0, \widetilde{E}_{n, q}(0)=\widetilde{E}_{n, q}$ is the $n$th $q$-Euler number with weight 0 (see $[5,11]$ ).

Throughout this paper, let $q$ be a complex number with $|q|<1$. As $q \rightarrow 1$, we obtain (1) and (2) from (3) and (4).
The generating function of Eulerian polynomial $H_{n}(x \mid$ $u$ ) is defined by

$$
\frac{1-u}{e^{t}-u} e^{x t}=\sum_{n=0}^{\infty} H_{n}(x \mid u) \frac{t^{n}}{n !}
$$

where $u \in \mathbb{C}$ with $u \neq 1$. In the special case, $x=0, H_{n}(0 \mid$ $u)=H_{n}(u)$ is called the $n$th Eulerian number (see [1-3]). Sometimes that is called the $n$th Frobenius-Euler number (see $[9-11,15])$.

From (1) and (5), we note that $H_{n}(x \mid-1)=E_{n}(x)$. From (5), we have

$$
H_{0}(u)=1, \quad H_{n}(1 \mid u)-u H_{n}(u)=(1-u) \delta_{0, n},
$$

where $\delta_{n, k}$ is Kronecker symbol (see [9-11]).

For $N \in \mathbb{N}$, the $q$-Euler polynomial of order $N$ is defined by the generating function as follows:

$$
\begin{aligned}
G_{q}^{N}(t, x) & =\underbrace{\left(\frac{2}{q e^{t}+1}\right) \times \cdots \times\left(\frac{2}{q e^{t}+1}\right) e^{x t}}_{N \text {-times }} \\
& =\sum_{n=0}^{\infty} \widetilde{E}_{n, q}^{(N)}(x) \frac{t^{n}}{n !} .
\end{aligned}
$$

In the special case, $x=0, \widetilde{E}_{n, q}^{(N)}(0)=\widetilde{E}_{n, q}^{(N)}$ is called the $n$th $q$-Euler number of order $N$ with weight 0 (see $[5,11]$ ). 
In [9], Kim derived some identities between the sums of products of Frobenius-Euler polynomials and FrobeniusEuler polynomials of higher order. The main idea is to construct nonlinear ordinary differential equations with respect to $t$ which are closely related to the generating function of Frobenius-Euler polynomial. In [3], Choi considered nonlinear ordinary differential equations with respect to $u$ not $t$.

In this paper, we construct nonlinear ordinary differential equations with respect to $q$. The purpose of this paper is to give some new identities on the high order $q$-Euler numbers and polynomials with weight 0 by using the differential equations of $q$.

\section{Construction of Nonlinear Differential Equations}

We define

$$
\begin{gathered}
G=G(q)=\frac{1}{q e^{t}+1}, \\
G^{N}(t, x)=\underbrace{G \times \cdots \times G}_{N \text {-times }} e^{x t} \text { for } N \in \mathbb{N} .
\end{gathered}
$$

From (7) and (8), we note that

$$
G_{q}^{N}(t, x)=2^{N} G^{N}(t, x)=2^{N} G^{N} e^{x t} .
$$

By differentiating (8) with respect to $q$, we get

$$
\begin{aligned}
& G^{(1)}=\frac{d G}{d q}=-\frac{q e^{t}+1-1}{q\left(q e^{t}+1\right)^{2}}=-\frac{G}{q}+\frac{G^{2}}{q}, \\
& q G^{(1)}+G=G^{2} .
\end{aligned}
$$

By differentiating (10) with respect to $q$, we get

$$
q^{2} G^{(2)}+4 q G^{(1)}+2 G=2 ! G^{3},
$$

where $G^{(N)}=d^{N} G / d q^{N}$.

By the derivative of (11) with respect to $q$, we have

$$
q^{3} G^{(3)}+9 q^{2} G^{(2)}+18 q G^{(1)}+3 ! G=3 ! G^{4} .
$$

Continuing this process, we get

$$
(N-1) ! G^{N}=\sum_{k=0}^{N-1} a_{k}(N) q^{k} G^{(k)} .
$$

Let us consider the derivative of (13) with respect to $q$ to find the coefficient $a_{k}(N)$ in (13).

By (10), we get

$$
\begin{aligned}
q \frac{d}{d q}\left((N-1) ! G^{N}\right) & =N ! G^{N-1} q G^{(1)} \\
& =N ! G^{N-1}\left(-G+G^{2}\right) \\
& =N ! G^{N+1}-N(N-1) ! G^{N} .
\end{aligned}
$$

From (13) and (14), we get

$$
\begin{aligned}
N ! G^{N+1}= & N(N-1) ! G^{N} \\
& +\sum_{k=0}^{N-1} k a_{k}(N) q^{k} G^{(k)} \\
& +\sum_{k=1}^{N} a_{k-1}(N) q^{k} G^{(k)},
\end{aligned}
$$

where $N ! G^{N+1}=\sum_{k=0}^{N} a_{k}(N+1) q^{k} G^{(k)}$.

By comparing coefficients on both sides of (15), we obtain the following recurrence relations:

$$
\begin{gathered}
a_{0}(N+1)=N a_{0}(N), \quad a_{N}(N+1)=a_{N-1}(N), \\
a_{k}(N+1)=N a_{k}(N)+k a_{k}(N)+a_{k-1}(N),
\end{gathered}
$$

for $1 \leq k \leq N-1$ and $a_{k}(N)=0$.

From the first part of (16), we have

$$
\begin{aligned}
a_{0}(N+1) & =N a_{0}(N) \\
& =N(N-1) a_{0}(N-1) \\
& =\cdots=N ! a_{0}(2) .
\end{aligned}
$$

By (10) and (13), we have

$$
\begin{aligned}
q G^{(1)}+G & =G^{2}=\sum_{k=0}^{1} a_{k}(2) q^{k} G^{(k)} \\
& =a_{0}(2) G+a_{1}(2) q G^{(1)} .
\end{aligned}
$$

From (18) and (19), we get

$$
a_{0}(2)=1, \quad a_{1}(2)=1, \quad a_{0}(N)=(N-1) ! .
$$

From the second part of (16), we have

$$
a_{N}(N+1)=a_{N-1}(N)=\cdots=a_{1}(2)=1 .
$$

To find $a_{k}(N)$ in (13) from (17), we set

$$
g(t, s)=\sum_{N \geq 1} \sum_{0 \leq k \leq N-1} a_{k}(N) \frac{t^{N}}{N !} s^{k},
$$

where $|t|<1$ (see [9]).

From (17) and (22), we have

$$
\begin{aligned}
\sum_{N \geq 1} & \sum_{0 \leq k \leq N-1} a_{k+1}(N+1) \frac{t^{N}}{N !} s^{k} \\
= & \sum_{N \geq 1} \sum_{0 \leq k \leq N-1} N a_{k-1}(N) \frac{t^{N}}{N !} s^{k} \\
& +\sum_{N \geq 1} \sum_{0 \leq k \leq N-1}(k+1) a_{k+1}(N) \frac{t^{N}}{N !} s^{k}+g(t, s) .
\end{aligned}
$$


From the left hand side of (23), we have

$$
\begin{aligned}
& \sum_{N \geq 1} \sum_{0 \leq k \leq N-1} a_{k+1}(N+1) \frac{t^{N}}{N !} s^{k} \\
& =\frac{1}{s} \sum_{N \geq 2} \sum_{1 \leq k \leq N-1} a_{k}(N) \frac{t^{N-1}}{(N-1) !} s^{k} \\
& =\frac{1}{s} \sum_{N \geq 2}\left(\sum_{0 \leq k \leq N-1} a_{k}(N) \frac{t^{N-1}}{(N-1) !} s^{k}-a_{0}(N) \frac{t^{N-1}}{(N-1) !}\right) \\
& =\frac{1}{s}\left(\sum_{N \geq 1} \sum_{0 \leq k \leq N-1} a_{k}(N) \frac{t^{N-1}}{(N-1) !} s^{k}-a_{0}(1)-\sum_{N \geq 2} t^{N-1}\right) \\
& =\frac{1}{s}\left(g_{t}+\frac{1}{t-1}\right),
\end{aligned}
$$

where $g_{t}=\partial g / \partial t$. From the first term of the right hand side of (23), we have

$$
\begin{aligned}
& \sum_{N \geq 1} \sum_{0 \leq k \leq N-1} N a_{k+1}(N) \frac{t^{N}}{N !} s^{k} \\
& =\frac{t}{s} \sum_{N \geq 1} \sum_{1 \leq k \leq N-1} a_{k}(N) \frac{t^{N-1}}{(N-1) !} s^{k} \\
& =\frac{t}{s}\left(\sum_{N \geq 1} \sum_{0 \leq k \leq N-1} a_{k}(N) \frac{t^{N-1}}{(N-1) !} s^{k}\right. \\
& =\frac{t}{s}\left(g_{t}+\frac{a_{0}(N)}{t-1}\right) .
\end{aligned}
$$

From the second term of the right hand side of (23), we have

$$
\begin{aligned}
\sum_{N \geq 1} & \sum_{0 \leq k \leq N-1}(k+1) a_{k+1}(N) \frac{t^{N}}{N !} s^{k} \\
& =\sum_{N \geq 1} \sum_{1 \leq k \leq N} k a_{k}(N) \frac{t^{N}}{N !} s^{k-1} \\
& =\sum_{N \geq 1} \sum_{0 \leq k \leq N-1} k a_{k}(N) \frac{t^{N}}{N !} s^{k-1}=g_{s},
\end{aligned}
$$

where $g_{s}=\partial g / \partial s$.

From (22)-(26), we obtain the following initial value problem quasilinear first-order partial differential equation:

$$
\begin{gathered}
(t-1) g_{t}+s g_{s}=-s g-1, \quad|t|<1, \\
g(0, s)=0, \quad s \in \mathbb{R} .
\end{gathered}
$$

We consider Cauchy problem for the following first-order quasilinear partial differential equation:

$$
\begin{aligned}
& P(x, y, z) z_{x}+Q(x, y, z) z_{y} \\
& \quad=R(x, y, z), \\
& z\left(x_{0}(t), y_{0}(t)\right)=z_{0}(t), \quad t \in I,
\end{aligned}
$$

where $I$ is some interval.

We know that (28) has a unique solution under some conditions as follows.

Theorem A (see [17, page 65]). Suppose that $P, Q$, and $R$ are of class $C^{1}$ in a domain $\Omega$ of $\mathbb{R}^{3}$ containing the point $\left(x_{0}, y_{0}, z_{0}\right)$ and suppose that

$$
P\left(x_{0}, y_{0}, z_{0}\right) \frac{d y_{0}\left(t_{0}\right)}{d t}-Q\left(x_{0}, y_{0}, z_{0}\right) \frac{d x_{0}\left(t_{0}\right)}{d t} \neq 0 \text {. }
$$

Then in a neighborhood $U$ of $\left(x_{0}, y_{0}\right)$ there exists a unique solution of (28) at every point of initial curve contained in $U$.

Since (27) satisfies (29) and regularity conditions, there exists a unique solution of (27).

It is customary to write (27) in the form

$$
\begin{aligned}
& \frac{d t}{t-1}=\frac{d s}{s}=\frac{d g}{-s g-1}, \\
& t=0, \quad s=p, \quad g=0 .
\end{aligned}
$$

Since $d t /(t-1)=d s / s$ is separable, we get

$$
u_{1}(t, s, g)=\frac{1-t}{s}
$$

$u_{1}$ is a solution of partial differential equation of (27).

From (30), we get the linear equation

$$
\frac{d g}{d s}=-g-\frac{1}{s} \text {. }
$$

By the integrating factor method, we have

$$
u_{2}(t, s, g)=e^{s} g+E_{i}(s)
$$

The exponential integral $E_{i}(s)$ is defined by

$$
\begin{aligned}
E_{i}(s) & =\int_{-\infty}^{s} \frac{e^{r}}{r} d r \\
& =\gamma+\ln |s|+\sum_{n=1}^{\infty} \frac{s^{n}}{n \cdot n !}, \quad(s \in \mathbb{R}, s \neq 0),
\end{aligned}
$$

where $\gamma$ is Euler constant.

$u_{2}$ is another solution of partial differential equation of (27), and $u_{1}$ and $u_{2}$ are linearly independent.

From the parameterized initial conditions (31), (33), and (34), we get

$$
u_{2}=E_{i}\left(\frac{1}{u_{1}}\right), \quad e^{s} g+E_{i}(x)=E_{i}\left(\frac{s}{1-t}\right) .
$$


Thus, from (35) and (36), we obtain the following unique solution of (27):

$$
g(t, s)=e^{-s}\left(-\ln |1-t|+\sum_{n=1}^{\infty} \frac{s^{n}}{n \cdot n !}\left(\left(\frac{1}{1-t}\right)^{n}-1\right)\right) .
$$

Moreover, if we choose another initial condition

$$
g(t, 0)=\sum_{N \geq 1}^{\infty} a_{0}(N) \frac{t^{N}}{N !}=\sum_{N \geq 1}^{\infty} \frac{t^{N}}{N}
$$

from (20) and (22), then (37) satisfies it.

We note that

$$
\begin{aligned}
\left(\frac{1}{1-t}\right)^{n}-1 & =\underbrace{\left(\sum_{l_{1} \geq 0} t^{l_{1}}\right) \times \cdots \times\left(\sum_{l_{n} \geq 0} t^{l_{n}}\right)}_{n \text {-times }}-1 \\
& =\sum_{N \geq 1}\left(\begin{array}{c}
\sum_{l_{1}+\cdots+l_{n}=N} t^{N}
\end{array}\right) \\
& =\sum_{N \geq 1}\left(\begin{array}{c}
n+N-1 \\
N
\end{array}\right) t^{N} .
\end{aligned}
$$

By (37) and (39), we get

$$
\begin{aligned}
g(t, s)= & \left(\sum_{k \geq 0} \frac{(-1)^{k}}{k !} s^{k}\right)\left(\sum_{N \geq 1} \frac{t^{N}}{N}\right) \\
& +\left(\sum_{k \geq 0} \frac{(-1)^{k}}{k !} s^{k}\right) \\
& \times\left(\sum_{n \geq 1} \frac{s^{n}}{n \cdot n !} \sum_{N \geq 1}\left(\begin{array}{c}
n+N-1 \\
N
\end{array}\right) t^{N}\right) \\
= & \sum_{N \geq 1} \sum_{0 \leq k \leq N-1} \frac{(-1)^{k}}{N \cdot k !} t^{N} s^{k}+\sum_{N \geq 1} \sum_{k \geq N} \frac{(-1)^{k}}{N \cdot k !} t^{N} s^{k} \\
& +\sum_{N \geq 1} \sum_{1 \leq k \leq N-1}\left(\sum_{l=1}^{k} \frac{(-1)^{k-l}}{(k-l) ! l \cdot l !}\left(\begin{array}{c}
l+N-1 \\
N
\end{array}\right) t^{N} s^{k}\right. \\
& +\sum_{N \geq 1} \sum_{k \geq N}\left(\sum_{l=1}^{k} \frac{(-1)^{k-l}}{(k-l) ! l \cdot l !}\left(\begin{array}{c}
l+N-1 \\
N
\end{array}\right) t^{N} s^{k} .\right.
\end{aligned}
$$

It is known that

$$
\begin{aligned}
(-1)^{l}\left(\begin{array}{c}
l+N-1 \\
N
\end{array}\right) & =\frac{l}{N}\left(\begin{array}{c}
-N \\
l
\end{array}\right), \\
\sum_{l=0}^{k}\left(\begin{array}{l}
k \\
l
\end{array}\right)\left(\begin{array}{c}
N \\
l
\end{array}\right) & =\left(\begin{array}{c}
k+N \\
k
\end{array}\right) .
\end{aligned}
$$

In the case of $k \geq N$ in (40), from (41), we get

$$
\begin{aligned}
\frac{(-1)^{k}}{N \cdot k !} & +\sum_{l=1}^{k} \frac{(-1)^{k-l}}{(k-l) ! l \cdot l !}\left(\begin{array}{c}
l+N-1 \\
N
\end{array}\right) \\
= & (-1)^{k}\left(\frac{1}{N \cdot k !}+\frac{1}{N \cdot k !} \sum_{l=1}^{k}\left(\begin{array}{l}
k \\
l
\end{array}\right)\left(\begin{array}{c}
-N \\
l
\end{array}\right)\right) \\
= & (-1)^{k} \frac{1}{N \cdot k !}\left(1+\left(\begin{array}{c}
k-N \\
k
\end{array}\right)-1\right)=0 .
\end{aligned}
$$

By (40) and (41), we get

$$
\begin{aligned}
g(t, s)= & \sum_{N \geq 1} \sum_{1 \leq k \leq N-1}(-1)^{k} \frac{(N-1) !}{k !}\left(\begin{array}{c}
k-N \\
k
\end{array}\right) \frac{t^{N}}{N !} s^{k} \\
& +\sum_{N \geq 1}(N-1) ! \frac{t^{N}}{N !} \\
= & \sum_{N \geq 1} \sum_{0 \leq k \leq N-1}(N-k-1) !\left(\begin{array}{c}
N-1 \\
k
\end{array}\right)^{2} \frac{t^{N}}{N !} s^{k},
\end{aligned}
$$

where $\left(\begin{array}{c}k-N \\ k\end{array}\right)=(-1)^{k}\left(\begin{array}{c}N-1 \\ k\end{array}\right)$. Thus, by (22) and (43), we get

$$
a_{k}(N)=(N-k-1) !\left(\begin{array}{c}
N-1 \\
k
\end{array}\right)^{2} .
$$

Therefore, by (13) and (44), we obtain the following theorem.

Theorem 1. For $q \in \mathbb{C}$ with $|q|<1$ and $N \in \mathbb{N}$, one can consider the following nonlinear $(N-1)$ th order ordinary differential equation with respect to q:

$$
\begin{aligned}
G^{N}(q) & =\frac{1}{(N-1) !} \sum_{k=0}^{N-1}(N-k-1) !\left(\begin{array}{c}
N-1 \\
k
\end{array}\right)^{2} q^{k} G^{(k)} \\
& =\sum_{k=0}^{N-1} \frac{1}{k !}\left(\begin{array}{c}
N-1 \\
k
\end{array}\right) q^{k} G^{(k)}
\end{aligned}
$$

where $G^{(k)}=d^{k} G^{(q)} / d q^{k}$ and $G^{N}(q)=\underbrace{G(q) \times \cdots \times G(q)}_{N \text {-times }}$. Then $G(q)=1 /\left(q e^{t}+1\right)$ is a solution of $(45)$.

Let us define $G^{(k)}(t, x)=G^{(k)}(q) e^{x t}$. Then we obtain the following corollary.

\section{Corollary 2. For $N \in \mathbb{N}$, one considers}

$$
\begin{aligned}
G^{N}(t, x) & =\frac{1}{(N-1) !} \sum_{k=0}^{N-1}(N-k-1) !\left(\begin{array}{c}
N-1 \\
k
\end{array}\right)^{2} q^{k} G^{(k)}(t, x) \\
& =\sum_{k=0}^{N-1} \frac{1}{k !}\left(\begin{array}{c}
N-1 \\
k
\end{array}\right) q^{k} G^{(k)}(t, x) .
\end{aligned}
$$

Then $G(t, x)=e^{x t} /\left(q e^{t}+1\right)$ is a solution of $(46)$. 


\section{Identities on the High-Order $q$-Euler Numbers and Polynomials with Weight 0}

From (3), (7), and (8), we get

$$
\begin{aligned}
G^{N}(q) & =\frac{1}{2^{N}} \underbrace{\left(\frac{2}{q e^{t}+1}\right) \times \cdots \times\left(\frac{2}{q e^{t}+1}\right)}_{N \text {-times }} \\
& =\frac{1}{2^{N}} \sum_{n=0}^{\infty} \widetilde{E}_{n, q}^{(N)} \frac{t^{n}}{n !}, \\
G(q) & =\frac{1}{2} \frac{2}{q e^{t}+1}=\frac{1}{2} \sum_{n=0}^{\infty} \widetilde{E}_{n, q} \frac{t^{n}}{n !} .
\end{aligned}
$$

From (47), we note that

$$
G^{(k)}=\frac{d^{k} G(q)}{d q^{k}}=\frac{1}{2} \sum_{n=0}^{\infty} \frac{d^{k} \widetilde{E}_{n, q}}{d q^{k}} \frac{t^{n}}{n !} .
$$

Therefore, by (47), (48), and (45), we obtain the following theorem.

Theorem 3. For $N \in \mathbb{N}$ and $n \in \mathbb{N} \cup\{0\}$, one has

$$
\widetilde{E}_{n, q}^{(N)}=2^{N-1} \sum_{k=0}^{N-1} \frac{1}{k !}\left(\begin{array}{c}
N-1 \\
k
\end{array}\right) q^{k} \frac{d^{k} \widetilde{E}_{n, q}}{d q^{k}} .
$$

From (48), we get

$$
\begin{aligned}
G^{(k)}(t, x) & =G^{(k)}(q) e^{x t} \\
& =\left(\frac{1}{2} \sum_{n=0}^{\infty} \frac{d^{k} \widetilde{E}_{n, q}}{d q^{k}} \frac{t^{n}}{n !}\right)\left(\sum_{n=0}^{\infty} \frac{x^{n} t^{n}}{n !}\right) \\
& =\sum_{n=0}^{\infty}\left(\sum_{l=0}^{n} \frac{1}{2}\left(\begin{array}{l}
n \\
l
\end{array}\right) x^{n-l} \frac{d^{k} \widetilde{E}_{l, q}}{d q^{k}}\right) \frac{t^{n}}{n !} .
\end{aligned}
$$

Therefore, by (7), (47), and (50), we obtain the following corollary.

Corollary 4. For $N \in \mathbb{N}$ and $n \in \mathbb{N} \cup\{0\}$, one has

$$
\widetilde{E}_{n, q}^{(N)}(x)=2^{N-1} \sum_{k=0}^{N-1} \frac{1}{k !}\left(\begin{array}{c}
N-1 \\
k
\end{array}\right) q^{k} \sum_{l=0}^{n}\left(\begin{array}{l}
n \\
l
\end{array}\right) x^{n-l} \frac{d^{k} \widetilde{E}_{l, q}}{d q^{k}}
$$

From (3) and (7), we get

$$
\begin{aligned}
\sum_{n=0}^{\infty} \widetilde{E}_{n, q}^{(N)} \frac{t^{n}}{n !} & =\underbrace{\left(\frac{2}{q e^{t}+1}\right) \times \cdots \times\left(\frac{2}{q e^{t}+1}\right)}_{N \text {-times }} \\
& =\left(\sum_{l_{1}=0}^{\infty} \widetilde{E}_{l_{1}, q} \frac{t^{l_{1}}}{l_{1} !}\right) \times \cdots \times\left(\sum_{l_{N}=0}^{\infty} \widetilde{E}_{l_{N}, q} \frac{t^{l_{N}}}{l_{N} !}\right)
\end{aligned}
$$

$$
\begin{aligned}
& =\sum_{n=0}^{\infty}\left(\sum_{l_{1}+\cdots+l_{N}=n} \frac{n ! \widetilde{E}_{l_{1}, q} \cdots \widetilde{E}_{l_{N}, q}}{l_{1} ! \cdots l_{N} !}\right) \frac{t^{n}}{n !} \\
& =\sum_{n=0}^{\infty}\left(\sum_{l_{1}+\cdots+l_{N}=n}\left(\begin{array}{c}
n \\
l_{1}, \ldots, l_{N}
\end{array}\right) \widetilde{E}_{l_{1}, q} \cdots \widetilde{E}_{l_{N}, q}\right) \frac{t^{n}}{n !} .
\end{aligned}
$$

Therefore, by (49) and (52), we obtain the following corollary.

Corollary 5. For $N \in \mathbb{N}$ and $n \in \mathbb{N} \cup\{0\}$, one has

$$
\begin{aligned}
& \sum_{l_{1}+\cdots+l_{N}=n}\left(\begin{array}{c}
n \\
l_{1}, \ldots, l_{N}
\end{array}\right) \widetilde{E}_{l_{1}, q} \ldots \widetilde{E}_{l_{N}, q} \\
& =2^{N-1} \sum_{k=0}^{N-1} \frac{1}{k !}\left(\begin{array}{c}
N-1 \\
k
\end{array}\right) q^{k} \frac{d^{k} \widetilde{E}_{n, q}}{d q^{k}} .
\end{aligned}
$$

\section{Acknowledgment}

The present research has been conducted by the research grant of Kwangwoon University in 2013.

\section{References}

[1] L. Carlitz, "Eulerian numbers and polynomials," Mathematics Magazine, vol. 32, pp. 247-260, 1959.

[2] L. Carlitz, "The product of two Eulerian polynomials," Mathematics Magazine, vol. 36, no. 1, pp. 37-41, 1963.

[3] J. Choi, "A note on Eulerian polynomials of higher order," Journal of the Chungcheong Mathematical Society, vol. 26, no. 1, pp. 191-196, 2013.

[4] J. Choi, T. Kim, and Y. H. Kim, "A recurrence formula for $q$ Euler numbers of higher order," Proceedings of the Jangjeon Mathematical Society, vol. 13, no. 3, pp. 321-326, 2010.

[5] J. Choi, T. Kim, and Y.-H. Kim, "A note on the $q$-analogues of Euler numbers and polynomials," Honam Mathematical Journal, vol. 33, no. 4, pp. 529-534, 2011.

[6] D. S. Kim, "Identities of symmetry for generalized Euler polynomials," International Journal of Combinatorics, vol. 2011, Article ID 432738, 12 pages, 2011.

[7] D. S. Kim, T. Kim, J. Choi, and Y. H. Kim, "Identities involving qBernoulli and q-Euler numbers," Abstract and Applied Analysis, vol. 2012, Article ID 674210, 10 pages, 2012.

[8] H.-M. Kim, J. Choi, and T. Kim, "On the extended $q$-Euler numbers and polynomials of higher-order with weight," Honam Mathematical Journal, vol. 34, no. 1, pp. 1-9, 2012.

[9] T. Kim, "Identities involving Frobenius-Euler polynomials arising from non-linear differential equations," Journal of Number Theory, vol. 132, no. 12, pp. 2854-2865, 2012.

[10] T. Kim and J. Choi, "A note on the product of Frobenius-Euler polynomials arising from the $p$-adic integral on $\mathbb{Z}_{p}$," Advanced Studies in Contemporary Mathematics, vol. 22, no. 2, pp. 215223, 2012.

[11] T. Kim and J. Choi, "On the $q$-Euler numbers and polynomials with weight 0," Abstract and Applied Analysis, vol. 2012, Article ID 795304, 7 pages, 2012.

[12] H. Ozden, I. N. Cangul, and Y. Simsek, "Multivariate interpolation functions of higher-order $q$-Euler numbers and their 
applications," Abstract and Applied Analysis, vol. 2008, Article ID 390857, 16 pages, 2008.

[13] H. Ozden and Y. Simsek, "A new extension of $q$-Euler numbers and polynomials related to their interpolation functions," Applied Mathematics Letters, vol. 21, no. 9, pp. 934-939, 2008.

[14] Y. Simsek, "Complete sum of products of $(h, q)$-extension of Euler polynomials and numbers," Journal of Difference Equations and Applications, vol. 16, no. 11, pp. 1331-1348, 2010.

[15] Y. Simsek, "Generating functions for q-Apostol type FrobeniusEuler numbers and polynomials," Axioms, vol. 1, no. 3, pp. 395403, 2012.

[16] Y. Simsek, "Generating functions for generalized Stirling type numbers, Array type polynomials, Eulerian type polynomials and their applications," Fixed Point Theory and Applications, vol. 2013, article 87, 2013.

[17] C. Zachmanoglou and D. W. Thoe, Introduction to Partial Differntial Equations with Applications, The Williams and Wilkins company, Baltimore, Md, USA, 1976. 


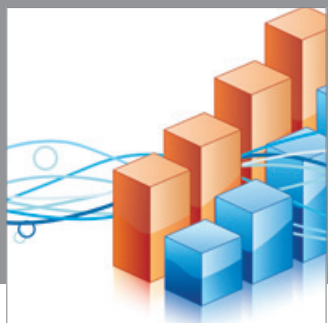

Advances in

Operations Research

mansans

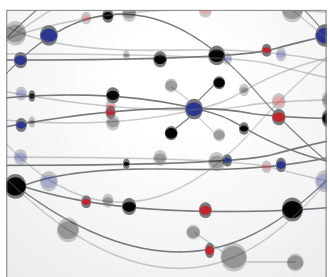

The Scientific World Journal
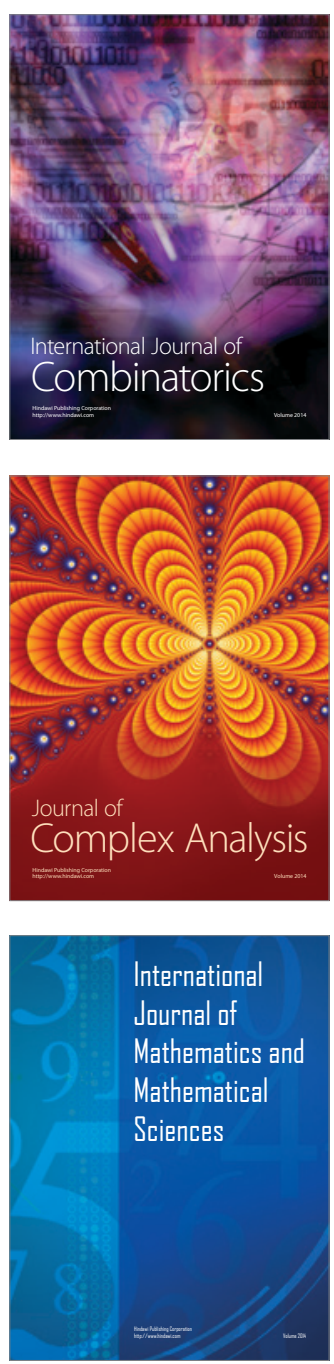
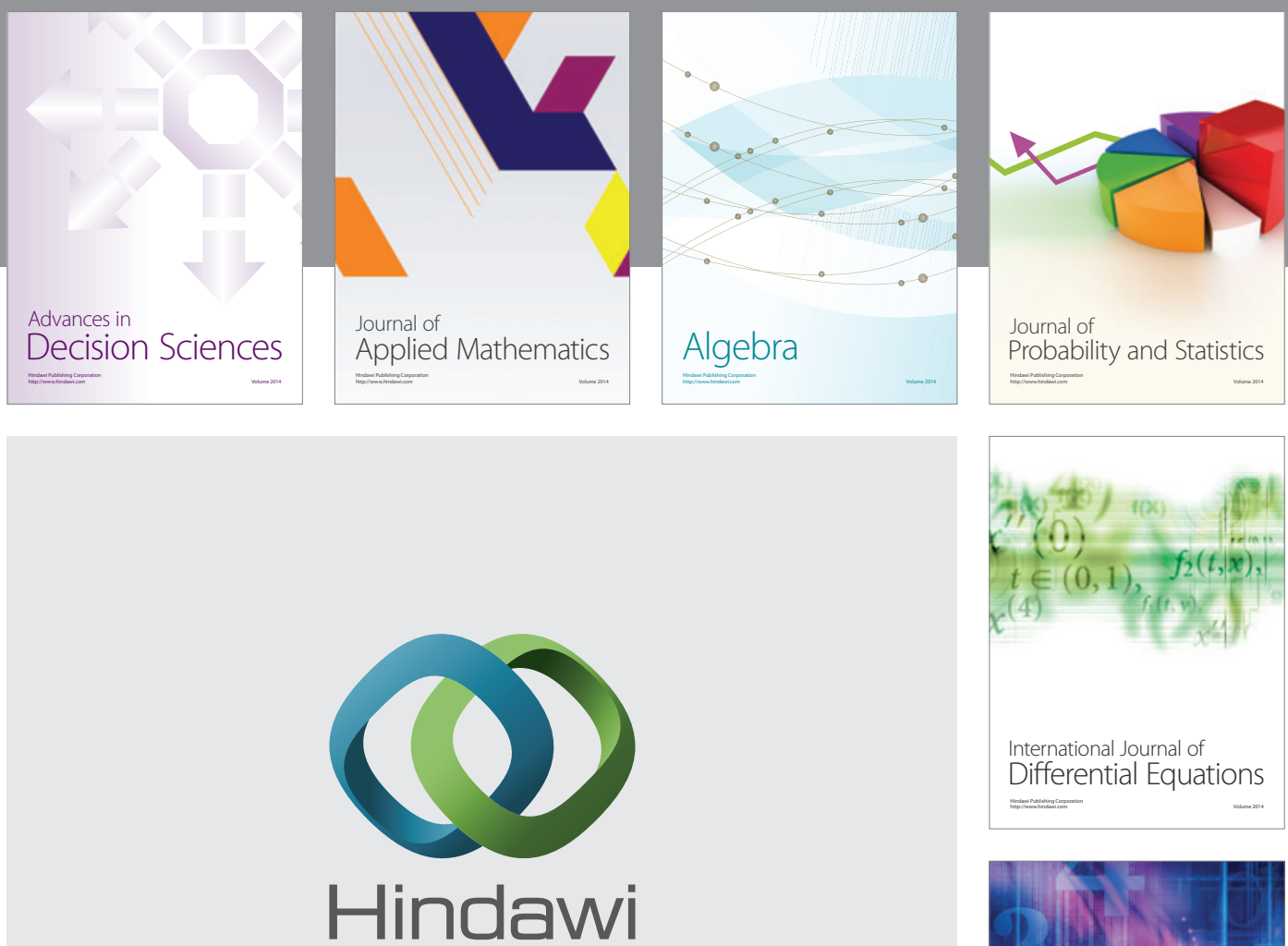

Submit your manuscripts at http://www.hindawi.com
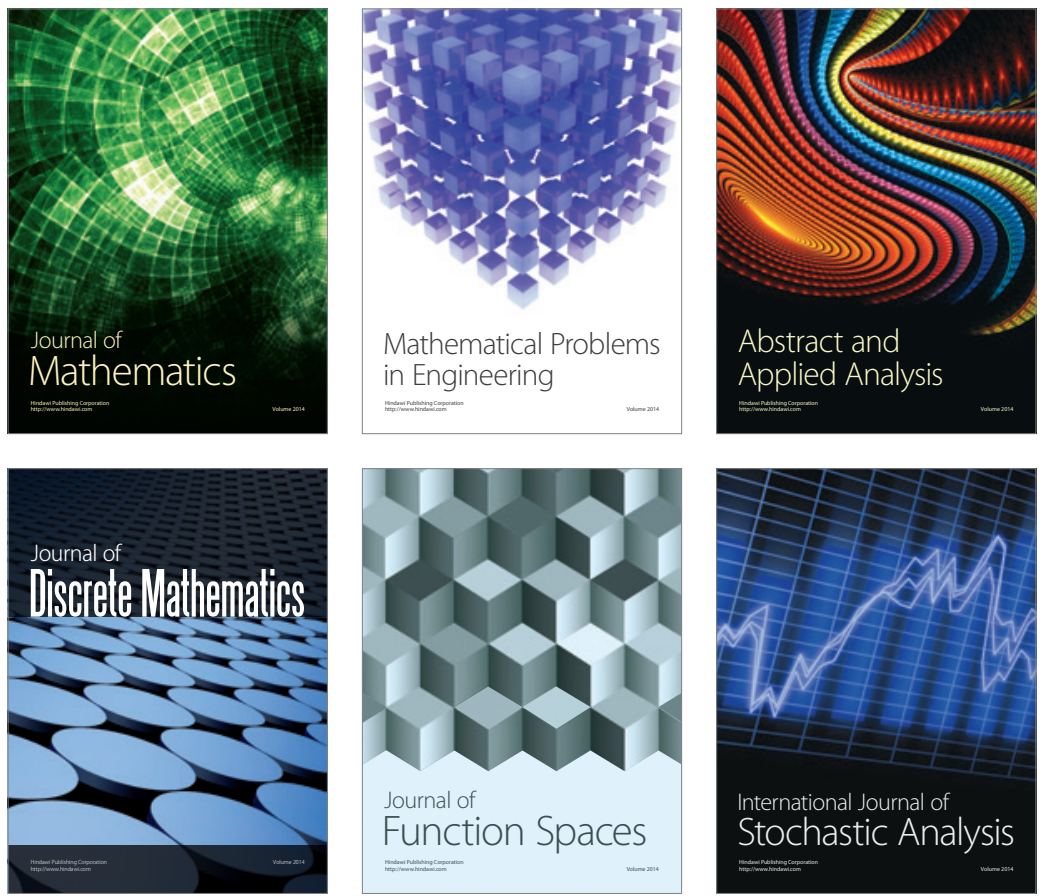

Journal of

Function Spaces

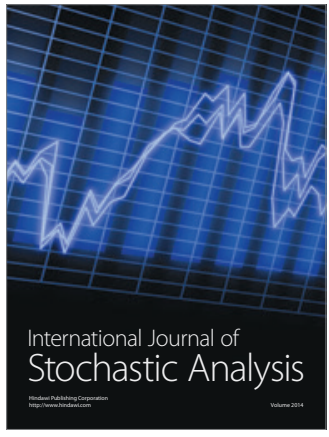

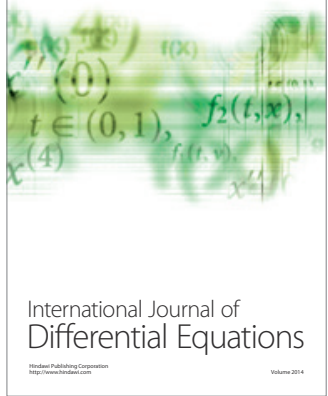
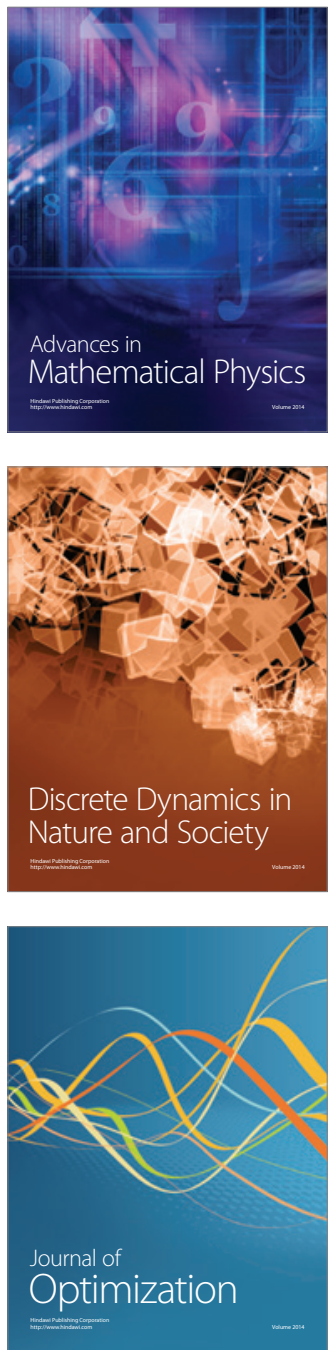